\title{
TOPOLOGY OF MILNOR FIBERS OF MINIMALLY ELLIPTIC SINGULARITIES
}

\author{
LEE J. McEWAN
}

(Communicated by Louis J. Ratliff, Jr.)

\begin{abstract}
Fundamental groups of Milnor fibers of some minimally elliptic singularities are computed via globalizations of smoothings. A conjecture of Looijenga-Wahl is verified for triangle singularities, which states that embedding dimension at most seven implies that any smoothing has simply-connected Milnor fiber.
\end{abstract}

Let $(X, p)$ be the two-dimensional germ of a minimally-elliptic (m.e.) singularity; we assume that $(X, p)$ belongs to one of the following three classes: simple-elliptic, cusp, or triangle singularity (their resolutions appear below). Let $M$ be the Milnor fiber of a smoothing of $(X, p)$. Questions about the topology of Milnor fibers in general are difficult to answer, beyond the rational or hypersurface case. This paper is concerned with the m.e. singularities named above. In particular, we show that a conjecture of Looijenga-Wahl is true in the case of triangle singularities: Embedding dimension $(X, p) \leq 7$ implies $M$ is simplyconnected. We also show that $\pi_{1}(M)$ is abelian for all triangle singularities to which our methods apply (about $75 \%$ of all values of $(p, q, r)$ for which $D_{p q r}$ has a smoothing) and to some smoothing components of some others. We also describe the topology of $M$ for simple-elliptic and cusp singularities and compute $\pi_{1}(M)$ in all cases of smoothings of simple-elliptic singularities. To some extent we show that $\pi_{1}(M)$ distinguishes smoothing components. We also provide proofs of statements made without proofs in [LW] concerning these examples.

\section{Globalizations and dual configurations}

In this section we explain the general set-up and basic facts that will be used. Our approach is to exploit the globalizations of smoothings of these singularities which were constructed by Looijenga.

Each of the three types can be realized on a complete surface which is conceived of as a neighborhood of the singularity with a configuration of curves glued on 'at infinity'; we call the latter the dual configuration, or just the dual. Except in the simple-elliptic case (which has a one-dimensional moduli given

Received by the editors September 30, 1991; preliminary version presented at a special session of an AMS-MAA meeting on August 10, 1990 in Columbus, OH.

1991 Mathematics Subject Classification. Primary 14J17; Secondary 14B07, 14J27, 14 F45. 
by the $j$-invariant), the topological data of the dual determines the singularity. For reference we list the graph of each resolution and dual configuration. In the diagrams that follow, a node represents a smooth rational curve and a number or a symbol denotes the self-intersection of that curve. Each edge represents a transverse intersection. (There are no triple points.)

(1.1) In type 1 the number of components in the dual equals $-C^{2}$.

(1.2) In type $2 d_{i}$ and $c_{j} \geq 2$ and some $d_{i_{0}}, c_{j_{0}} \geq 3$. If $n$ (or $m$ ) equals 1 , the configuration is a rational curve with an ordinary double point and has negative self-intersection. There is an algorithm for obtaining one configuration from the other [L].

(1.3) In types 1 and 2 the dual configuration $D$ is a negative anticanonical divisor: $-D \in|K|$. We refer briefly to such a configuration as an anticanonical cycle.

(1.4) In type 3 we require $1 / p+1 / q+1 / r<1$ and suppose $r \geq q \geq p \geq 2$. The dual configuration labeled $T_{p q r}$, has arms of $p, q$, and $r$ nodes, counting from the center.

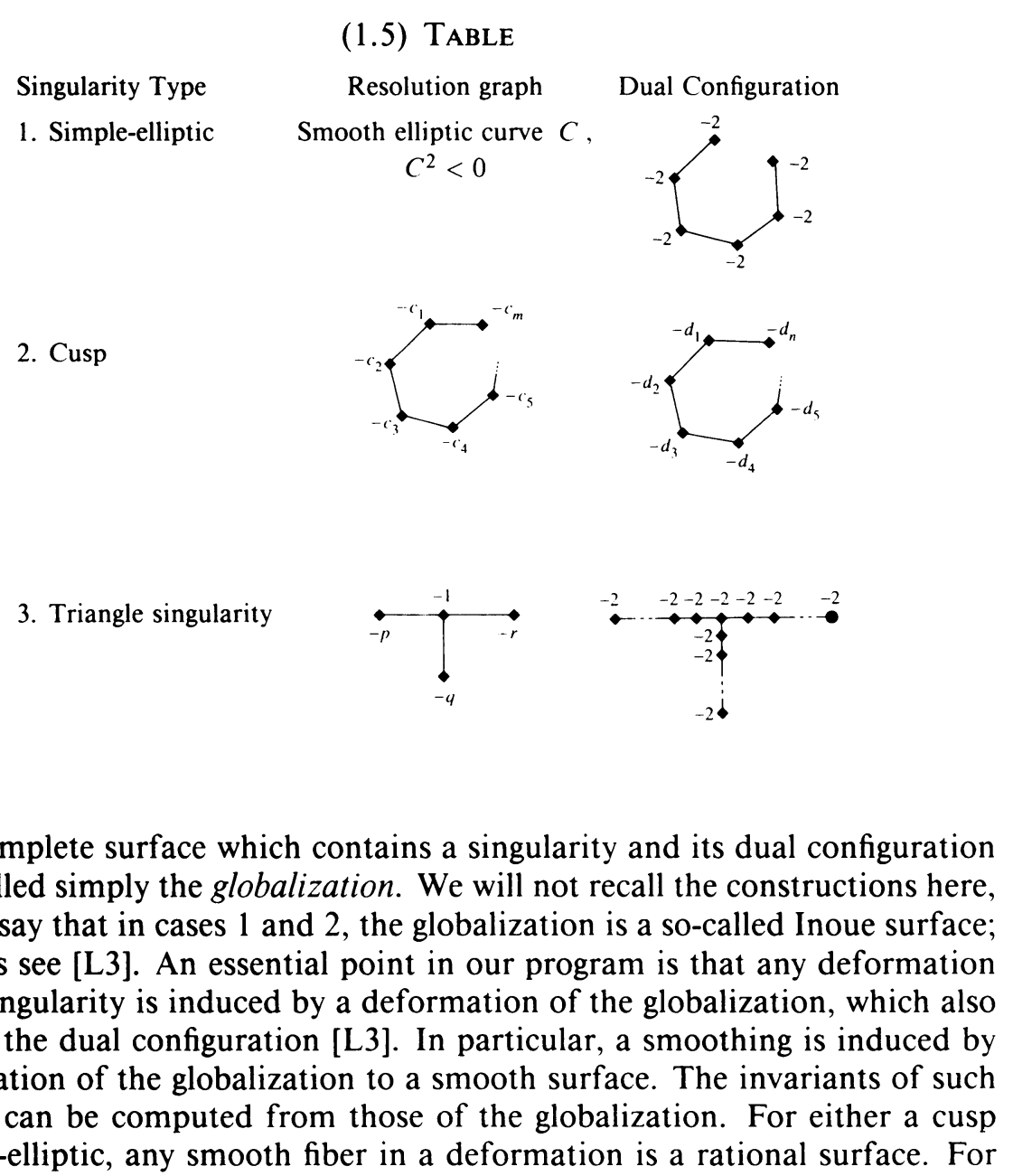

The complete surface which contains a singularity and its dual configuration will be called simply the globalization. We will not recall the constructions here, except to say that in cases 1 and 2, the globalization is a so-called Inoue surface; for details see [L3]. An essential point in our program is that any deformation of each singularity is induced by a deformation of the globalization, which also preserves the dual configuration [L3]. In particular, a smoothing is induced by a deformation of the globalization to a smooth surface. The invariants of such a surface can be computed from those of the globalization. For either a cusp or simple-elliptic, any smooth fiber in a deformation is a rational surface. For 
a triangle singularity, a smooth fiber is a K3 surface. In each case the smooth fiber contains the dual configuration and the Milnor fiber is just the complement of the dual.

In the case of simple-elliptic and triangle singularities, there is a complete converse: the existence of a smooth surface as described above implies that the corresponding singularity has a smoothing. Any such abstractly given surface is isomorphic to a fiber in the versal deformation of the (globalized) singularity. These results appear in [L1, L2]. The analogous statement for cusps is known as Looijenga's conjecture.

\section{TRIANGLE SINGULARITIES}

Let $D_{p q r}$ denote the triangle singularity corresponding to the values $(p, q, r)$. To construct a smoothing, we need only find a $\mathrm{K} 3$ surface which possesses a dual configuration, i.e., a $T_{p q r}$ curve. The following facts $((2.1)-(2.7))$ are stated in [LW].

(2.1) $D_{p q r}$ has a smoothing iff $p+q+r \leq 22$ and $(p, q, r) \neq(2,10,10)$ [P]. (There are 187 values of $(p, q, r)$ satisfying (1.4) and these two conditions.)

(2.2) The set $\operatorname{Sm}\left(D_{p q r}\right)$ of smoothing components of $D_{p q r}$ corresponds bijectively to $\zeta\left(D_{p q r}\right)$ (= the set of smoothing data of $\left.D_{p q r}\right)$.

(2.3) Let $N\left(D_{p q r}\right)$ be a regular neighborhood of $D_{p q r}$, and let $L=\partial N\left(D_{p q r}\right)$ be the boundary of its closure; $L$ is the 'link' of the singularity, diffeomorphic to the boundary of a Milnor fiber of any smoothing, i.e., $L \approx \partial N\left(T_{p q r}\right)$ where $N\left(T_{p q r}\right)$ is a regular neighborhood of a $T_{p q r}$ curve on a $\mathrm{K} 3$ surface. Then the first integral homology group $H_{1}(L ; \mathbb{Z})$ is finite and has the structure of a quadratic group; to conform with the notation of [LW], we will write this group as $H_{1}(L)_{t}$ ( $t$ indicates torsion subgroup). Any smoothing determines an isotropic subgroup $I$ of $H_{1}(L)_{t} . I$ is part of the 'smoothing data' which are the elements of $\zeta\left(D_{p q r}\right)$.

(2.4) By [LW, (4.18) and Table 1], $p+q+r<17$ implies:

(1) $I=0$ is the only isotropic subgroup; and

(2) I determines the other smoothing data.

(2.5) Combining the above statements, $p+q+r<17$ implies that $D_{p q r}$ has a unique smoothing component.

(2.6) Part (2) of (2.4) is also true when $p+q+r=17,18,19$ and when $p+q+r=20,21$ and $I^{\perp} / I$ is cyclic. $H_{1}(L)_{t}$ is cyclic exactly when $(p, q, r)$ has no common divisors. Thus there are unique smoothing components in several cases where $17 \leq p+q+r \leq 21$ and no nontrivial $I$ occur.

(2.7) The embedding dimension of $D_{p q r}$ is given as follows [LW, (6.1.1) and (6.5)]:

$$
\text { Emb. } \operatorname{dim}\left(D_{p q r}\right)=\left\{\begin{array}{lll}
r-6, & (p, q, r)=(2,3, r) & r \geq 7, \\
q+r-8, & (p, q, r)=(2, q, r) & q \geq 4, \\
p+q+r-9, & & p \geq 3 .
\end{array}\right.
$$

(In cases where these formulas give less than 3 , the embedding dimension is 3 .) 
(2.8) Next we construct K3 surfaces with $T_{p q r}$ curves on them. We have two methods for doing this, both of which involve elliptic fibrations. In what follows we use the results and notation of Kodaira [K, II, p. 565] concerning elliptic fibrations. The following lemma may be found in [SI].

(2.9) Lemma. Assume $D$ is an effective divisor on a K3 surface $X$ and that $D$ has the same type as a simple singular fiber of an elliptic surface (call such $D$ a Kodaira singular fiber). Then there exists a unique elliptic pencil $\phi: X \rightarrow \mathbb{P}^{1}$ such that $D$ is a singular fiber. Any irreducible curve $C$ such that $(C \cdot D)=1$ defines a section of $\phi$.

(2.10) Now suppose $X$ is a K3 surface containing a $T_{p q r}$ curve. We can locate a Kodaira singular fiber inside the $T_{p q r}$ of type $\mathrm{II}^{*}$, III ${ }^{*}$, or $\mathrm{IV}^{*}$, depending on the values of $p, q$, and $r$. Specifically, let $D$ be the divisor defined by one of the following diagrams, where the integers denote multiplicities of the components in $D$ (each $D$ is contained set-wise in a $\left.T_{p q r}\right)$ :

(a)

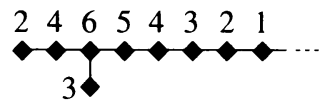

(b)
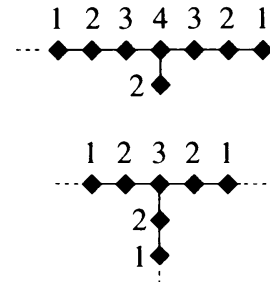

$p=2, q=3, r \geq 7 ; D$ is a $\mathrm{II}^{*}$.

$p=2, q \geq 4 ; D$ is a III* $^{*}$

$p \geq 3 ; D$ is a $\mathrm{IV}^{*}$

By Lemma (2.9), $D$ must induce an elliptic pencil on $X$, and the other components of the $T_{p q r}$ curve can be analyzed in terms of this fibration. It is easily checked that every component $C$ of the $T_{p q r}$, regarded as an effective divisor, satisfies $(D \cdot C)=0$ or 1 , with $(D \cdot C)=1$ exactly for the components adjacent to $D$. By Lemma (2.9) these components define sections of $\phi$.

(2.11) Lemma. Let $\phi: X \rightarrow \mathbb{P}^{1}$ be an elliptic surface, $E$ a reducible curve on $X$ such that $E$ contains at most one complete fiber $(E$ may contain components of several fibers), and $N$ the complement of $E$ on $X$. Let $f$ be any nonmultiple fiber of $\phi$ (smooth or singular) which contains no component of $E$. Let $f^{\prime}=f \backslash(f \cap E)$ and $i: f^{\prime} \rightarrow N$ be the inclusion. Then the induced map on fundamental groups is a surjection: $i_{*}: \pi_{1}\left(f^{\prime}\right) \rightarrow \pi_{1}(N)$.

Proof. Fix $f$ as in the hypothesis, and let $\gamma$ be a loop in $N$ representing $[\gamma] \in \pi_{1}(N)$. We take the base point of $\pi_{1}(N)$ to be on $f$. Let $p=\phi(f)$ and let $\left\{p_{i}\right\}_{i=0}^{n}$ be the points in $\mathbb{P}^{1}$ which are the images of the components of $E$ that belong to fibers of $\phi$, with $p_{0}$ the image of the only complete fiber in $E$. Then $\phi(\gamma)$ lies in $\mathbb{P}^{1} \backslash\left\{p_{0}\right\}$. We wish to homotope $\gamma$ in $N$ onto $f^{\prime}$, i.e., we want a homotopy of $\gamma$ to a loop $\bar{\gamma}$ with $\phi(\bar{\gamma})=p$. $\gamma$ can be contracted above $\mathbb{P}^{1} \backslash\left\{p_{0}\right\}$ until $\phi(\gamma)$ reaches a $p_{i}, i>0$. By assumption $\phi^{-1}\left(p_{i}\right)$ contains a component which does not belong to $E$. Let $\Delta$ be the image of a local topological section to $\phi$ which passes through this component and is contained in $N$. Then $\phi(\partial \Delta)$ is a loop around $p_{i}$. Since $\phi^{-1}(\phi(\Delta))$ is connected, we can homotope a portion of $\gamma$ so that it runs along part of the boundary of $\Delta$ and then across $\Delta$ to move over $p_{i}$. Doing this for each $p_{i}, i>0$, we can eventually homotope $\gamma$ to $\bar{\gamma}$, a loop supported on $f^{\prime}$. Then $i_{*}([\bar{\gamma}])=[\gamma]$. 


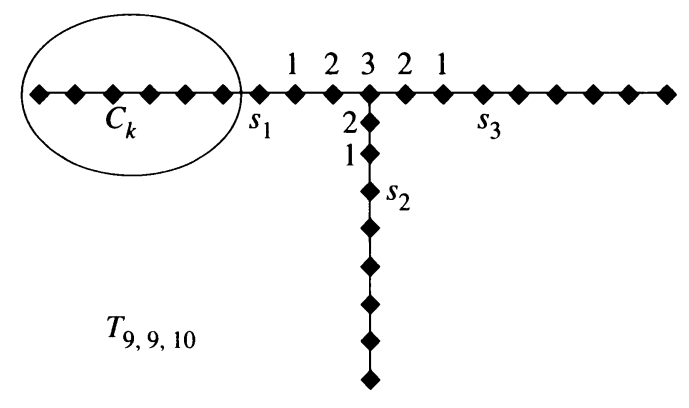

(2.12) We will apply this lemma to a $\mathrm{K} 3$ surface $X$ with a $T_{p q r}$. Such an $X$ is an elliptic surface with the divisor $D$ as a (complete) singular fiber. The components of $T_{p q r}$ adjacent to $D$ are sections of $\phi$, by Lemma 2.10. The connected chain of components on the other side of each section must belong to a single fiber of $\phi$; for a concrete example, consider the following $T_{9,9,10}$ diagram. The divisor $D$ (a IV* fiber in Kodaira's notation) consists of the central seven components with indicated multiplicities. The adjacent components are labeled $s_{i}, i=1,2,3$; they must be sections. The remainder of each arm (one is circled in the drawing above) must belong to a single fiber of the elliptic pencil; this is because each component $C_{k}$ is disjoint from $D$, and so cannot meet the generic fiber, i.e., $\phi\left(C_{k}\right)$ is a point. Since the chain is connected, each component maps to the same point.

By the classification of singular fibers, a complete fiber cannot be constructed out of the three arms alone; there must be extra components not belonging to the $T_{p q r}$. Therefore, $D$ is the only complete fiber of $\phi$ whose components all belong to the $T_{p q r}$. Consequently, the $T_{p q r}$ curve satisfies the hypothesis of Lemma (2.11), with $T_{p q r}$ as the curve $E$.

(2.13) The following three cases are most easily examined: $(p, q, r)=$ $(2,3, r),(2,4, r)$, and $(3,3, r)$. Applying (1.4) and (2.1), these three cases account for 34 smoothable triples. In the situation of $(2.12)$, there is only one section of $\phi$ in the curve $T_{p q r}$ in any of these cases (the divisor $D$ is at one end of the configuration). In the notation of Lemma (2.11) $f^{\prime}=f \backslash\left(f \cap T_{p q r}\right)$ is a once-punctured fiber. If $f$ is any singular fiber, then by the classification of singular fibers, $\pi_{1}\left(f^{\prime}\right)$ is abelian. Since the Milnor fiber $M$ of $D_{p q r}$ is just $X \backslash T_{p q r}$, we have by Lemma (2.11) that $\pi_{1}(M)$ is a quotient of $\pi_{1}\left(f^{\prime}\right)$, so $\pi_{1}(M)$ is abelian. We need only show that any pencil $\phi$ constructed as above must contain singular fibers with no components in common with $T_{p q r}$. We will do this for the case $T_{23 r}$; the other two cases follow exactly the same way. Suppose a K3 surface $X$ with a $T_{23 r}$ is given, and the elliptic pencil $\phi$ has only two singular fibers: $D$ (a II* fiber) and the fiber $F$ which contains the $r-7$ components adjacent to the sole section. We are in the case of $(2.10 \mathrm{a})$. It is well known that the euler characteristic of an elliptic fibration over $\mathbb{P}^{1}$ is equal to the sum of the euler characteristics of the singular fibers $[\mathrm{K}]$. Since $X$ is a K3, $e(X)=24$. Then since $e\left(\mathrm{II}^{*}\right)=10$, we must have $e(F)=14$. By the classification of singular fibers, either $F=I_{14}$ or $F=I_{8}^{*}$. In either case, the Picard number of $X$ is now too big: $D$ contributes eight independent elements to the Neron-Severi group in addition to the section and the class of a smooth 
fiber; an $I_{14}$ would contribute 13 additional independent elements, and an $I_{8}^{*}$ would contribute 12 , giving the group 22 or 23 independent generators. But the Picard number of $X$ is at most 20. (See [S, Theorem 1.1].) We have proven the following

(2.14) Proposition. Let $M$ be the Milnor fiber of any smoothing of the triangle singularity $D_{p q r}$, where $(p, q, r)=(2,3, r),(2,4, r)$, or $(3,3, r)$. Then $\pi_{1}(M)$ is abelian (and cyclic).

(2.15) In the last paragraph we considered a natural elliptic pencil carried by a $\mathrm{K} 3$ with a $T_{p q r}$; another approach is to construct an elliptic K3 surface directly and then find a $T_{p q r}$ curve on it. Constructions of a special type of such surfaces were given by Miranda and Persson [MP]; their program was to construct all elliptic K3 surfaces which possess only $I_{n}$ singular fibers ("semi-stable fibrations"). In particular, they were able to give a complete list of all combinations of $I_{n}$ singular fibers which can occur in an elliptic K3; the members of the list do occur, and any combination not on the list cannot occur. A combination is a set $\left\{I_{n_{1}}, \ldots, I_{n_{s}}\right\}$ of $I_{n}$ fibers and is referred to by the tuple of positive integers $\left[n_{1}, \ldots, n_{s}\right]$; by reasoning with the Picard number as above, we must have $s \geq 6$, and $\sum n_{i}=24$ by the Euler characteristic. All of their examples are algebraic and so possess a section $s$. We may find a $T_{p q r}$ on such a surface as follows. Select three of the $I_{n}$ fibers (and write them as $I_{a}, I_{b}$, and $I_{c}$ ) and the section. From each of the three $I_{n}$ fibers, drop one of the components which is adjacent to the component that meets the section. What remains is a $T_{p q r}$ configuration, with $(p, q, r)=(a, b, c)$ (in some order); the central component of the $T_{p q r}$ is the section $s$.

(2.16) The $T_{p q r}$ constructed above satisfies Lemma (2.11); it also has the same properties as the fibrations considered in Proposition (2.14); namely, the generic fiber meets $T_{p q r}$ in only one point, and there are singular fibers no component of which belongs to the $T_{p q r}$ (the $s-3 \geq 3$ remaining $I_{n}$ fibers). Therefore, reasoning as we did for Proposition (2.14), any K3 surface $X$ and $T_{p q r} \subset X$ constructed by this method satisfies the conclusion of the proposition: $\pi_{1}\left(X \backslash T_{p q r}\right)=\pi_{1}(M)$ is abelian and cyclic.

The following is a partial list of the 6-tuples which correspond to constructible configurations of $I_{n}$ fibers on elliptic K3 surfaces (the list is copied out of the complete list in [MP, Theorem 3.1]). The sum of the last three integers in each tuple is 21 ; following the outline of (2.15), each tuple gives a $T_{p q r}$ with $p+q+r=21$. All but six such smoothable triples appear in the list.

$\begin{array}{llll}{[1,1,1,2,3,16]} & {[1,1,1,2,4,15]} & {[1,1,1,2,5,14]} & {[1,1,1,2,6,13]} \\ {[1,1,1,2,7,12]} & {[1,1,1,2,8,11]} & {[1,1,1,2,9,10]} & {[1,1,1,3,3,15]} \\ {[1,1,1,3,4,14]} & {[1,1,1,3,5,13]} & {[1,1,1,3,6,12]} & {[1,1,1,3,7,11]} \\ {[1,1,1,3,8,10]^{*}} & {[1,1,1,4,6,11]^{*}} & {[1,1,1,4,7,10]^{*}} & {[1,1,1,5,5,11]} \\ {[1,1,1,5,6,10]} & {[1,1,1,5,7,9]^{*}} & {[1,1,1,6,7,8]} & {[1,1,1,7,7,7]}\end{array}$

The table is arranged lexicographically from left to right. Asterisks indicate gaps where smoothable triples $(p, q, r)$ would occur, but no elliptic pencil exists in the list. Explicitly, the following are the only triples missing from the list: $[1,1,1,3,9,9],[1,1,1,4,4,13],[1,1,1,4,5,12],[1,1,1,4,8,9]$, $[1,1,1,5,8,8],[1,1,1,6,6,9]$. We can also obtain $T_{p^{\prime} q^{\prime} r^{\prime}}$ configurations 
whenever $\left(p^{\prime}, q^{\prime}, r^{\prime}\right) \leq(p, q, r)$ and $(p, q, r)$ appears, by dropping some components from $T_{p q r}$. Thus from the list above, we can obtain a $T_{p^{\prime} q^{\prime} r}$ for all values of $\left(p^{\prime}, q^{\prime}, r^{\prime}\right)$ with $p^{\prime}+q^{\prime}+r^{\prime}=20$ except the following: [4, 4, 12], $[4,8,8]$. These can be constructed from other entries in Miranda-Persson's list: $[1,1,2,4,4,12]$ and $[1,1,2,4,8,8]$ also occur. Using the last three integers in each tuple as before, we can construct the missing values. We collect these observations as

(2.17) Lemma. For any $(p, q, r)$ satisfying (2.1) and (1.4) and $p+q+r \leq$ 21 with the possible exception of $(3,9,9),(4,4,13),(4,5,12),(4,8,9)$, $(5,8,8)$, and $(6,6,9)$, there exists a $K 3$ surface having a $T_{p q r}$ curve such that $\pi_{1}\left(X \backslash T_{p q r}\right)$ is abelian and cyclic.

Remark. This shows that at least one smoothing component of $D_{p q r}$ has a Milnor fiber with abelian $\pi_{1}$ for every value of $(p, q, r)$ for which the singularity has smoothings except for 35 cases, three of which are covered by Proposition (2.14). Most of these cases are at the upper limit: $p+q+r=22$.

(2.18) Proposition. Emb. $\operatorname{dim}\left(D_{p q r}\right) \leq 7$ implies $\pi_{1}(M)$ is abelian where $M$ is the Milnor fiber of any smoothing.

Proof. If $p>2$ then $\operatorname{Emb} \operatorname{dim}\left(D_{p q r}\right)=p+q+r-9$ by (2.7). Then Emb. $\operatorname{dim}\left(D_{p q r}\right) \leq 7$ is equivalent to $p+q+r \leq 16$; by $(2.5) D_{p q r}$ has a unique smoothing component, and by Lemma (2.17) there exists a Milnor fiber with abelian $\pi_{1}$. If $p=2$ and $q=3$ or 4 then all smoothings have abelian $\pi_{1}$ by Proposition (2.14). There remains the case $p=2, q>4$. By (2.5) again, Emb. $\operatorname{dim}\left(D_{p q r}\right)=q+r-8 \leq 7$ implies $p+q+r=2+q+r \leq 17$. If $p+q+r<17$, we argue as before that $D_{p q r}$ has a unique smoothing component and some fiber has an abelian $\pi_{1}(M)$. Suppose $p+q+r=17$. There are only three such triples: $(2,5,10),(2,6,9)$, and $(2,7,8)$. None of these occur in the list of [LW] (see (2.4)-(2.6)), i.e., each of the singularities given by these triples has a unique smoothing component and the same argument applies again.

Actually it is easy to see from the list in [LW] that the smoothing data is unique in all cases of this proposition. It then follows from [LW] that $\pi_{1}(M)=H_{1}(M)=H_{1}(L)_{t} / I^{\perp}=0$. This can also be proved directly from the construction of the K3 surfaces used in Lemma (2.17). Thus:

(2.19) Corollary. Emb. $\operatorname{dim}\left(D_{p q r}\right) \leq 7$ implies any Milnor fiber of any smoothing is simply connected.

\section{CUSPS AND SIMPLE-ELLIPTICS}

Let $(X, p)$ be a cusp or simple-elliptic singularity. Then any smoothing globalizes to a rational surface, with dual configuration an anticanonical cycle (see (1.3)). We will employ the usual practice of referring to the cycle by the sequence of negative self-intersections of its components: $\left[d_{1}, \ldots, d_{n}\right]$ stands for the cycle with $n$ components $\left\{D_{i}\right\}$ with $D_{i}^{2}=-d_{i}$. The ordering of the sequence is determined by $D$ up to a cyclic permutation or reversal. Of course, for a simple-elliptic every $d_{i}$ equals 2 , and only the length $n$ is important. The topology of rational surfaces with anticanonical cycles was analyzed in [M]. We apply the results of that analysis. 
(3.1) Emb. $\operatorname{dim}(X, p)=\max (3, n)$ where $n$ is the length of $D$.

The following proposition is due to Miranda; see [M] for a proof.

(3.2) Proposition. Suppose $(Y, D)$ is a rational surface with anticanonical cycle $D=\left[d_{1}, \ldots, d_{n}\right]$, with $n \geq 3$. Then $Y$ is obtained by blowing up $\mathbb{P}^{2}$ on three general lines.

In other words we can think of $Y$ as obtained from $\mathbb{P}^{2}$ by blowing up on the triangle formed by the three standard coordinate lines $x_{i}=0 \quad(i=$ $0,1,2)$, where $\left[x_{0}: x_{1}: x_{2}\right]$ are homogeneous coordinates. It is convenient to arrange the blowing up into two sequences. The first sequence consists of all blowups at nodes of the cycle ("corner blowups"); the second sequence consists of all blowups away from nodes, i.e., on smooth points of the cycle ("interior blowups"). Let $\bar{Y}$ be the intermediate surface obtained after performing all the corner blowups. $\bar{Y}$ has an anticanonical cycle of the same length as that of $Y$. Let $\bar{D}=\left[\bar{d}_{1}, \ldots, \bar{d}_{n}\right]$ denote the cycle of $\bar{Y}$. (Then $\bar{d}_{i} \leq d_{i}$, with equality for components with no interior blowups.) The complement of $\bar{D}$ is isomorphic to the complement of the triangle in $\mathbb{P}^{2}$, which can be viewed as $\mathbb{C}^{*} \times \mathbb{C}^{*}$ (where $\mathbb{C}^{*}$ is the complex numbers minus zero). The space $Y \backslash D$ can be understood topologically as the result of attaching two-handles to $\bar{Y} \backslash \bar{D}$; each interior blowup contributes one two-handle.

(3.3) It is important to note that the sequence of blowups which realize a given cycle $D$ is not unique; different sequences yield a different $Y$ and $\bar{Y}$ in general, with possibly different $\pi_{1}(M)$. In fact, different smoothing components of the singularity may be detected this way.

(3.4) Since $\bar{Y} \backslash \bar{D}$ is isomorphic to $\mathbb{C}^{*} \times \mathbb{C}^{*}, \pi_{1}(\bar{Y} \backslash \bar{D}) \quad(=\mathbb{Z} \oplus \mathbb{Z})$ is abelian. Attaching two-handles to a topological space in general either has no effect on $\pi_{1}$ or kills some classes. Therefore, $\pi_{1}(Y \backslash D)$ is always abelian. From [GS, Theorem 2, p. 540], $b_{1}$ (the first betti number of $Y \backslash D$ ) is zero; it follows that $\pi_{1}$ is finite.

(3.5) Let $N(\bar{D})$ be a regular neighborhood of $\bar{D}$ in $\bar{Y}$, and let $(\bar{Y} \backslash N(\bar{D}))^{\mathrm{c}}$ be the closure of the complement of $N(\bar{D})$. Then the boundary of $(\bar{Y} \backslash N(\bar{D}))^{\mathrm{c}}$ is diffeomorphic to the product of three circles: $\partial(\bar{Y} \backslash N(\bar{D}))^{\mathrm{c}} \approx S^{1} \times S^{1} \times S^{1}$. One factor of the product is homotopically trivial in $(\bar{Y} \backslash N(\bar{D}))^{\mathrm{c}}$.

It is natural to regard this three-manifold boundary as a (trivial) torus-bundle over $S^{1}$, in which the homotopically-trivial cycle appears as a section of the bundle. (Think of a generator of $H_{1}(\bar{D})$.) We may speak of 1-cycles supported in fibers of this bundle. Then the attaching spheres of the two-handles occurring in the interior blowups are all 1-cycles supported in fibers. We may suppose henceforth (via a cyclic permutation of the labeling, if necessary) that $\bar{d}_{1}<d_{1}$; this simply means that $\bar{D}_{1}$ has interior blow-ups occurring on it. Let $m$ be the number of components which receive internal blow ups and $i_{j}$ be the index of the $j$ th such, $j=1, \ldots, m$. (So, e.g., $i_{1}=1 ; \bar{d}_{i_{2}}<d_{i_{2}}$ and $\bar{d}_{i}=d_{i}$ for $1<i<i_{2}$.)

(3.6) Define:

$$
\left(\begin{array}{ll}
a_{i} & b_{i} \\
c_{i} & d_{i}
\end{array}\right):= \begin{cases}\left(\begin{array}{ll}
\bar{d}_{i-1} & 1 \\
-1 & 0
\end{array}\right)\left(\begin{array}{cc}
\bar{d}_{i-2} & 1 \\
-1 & 0
\end{array}\right) \cdots\left(\begin{array}{ll}
\bar{d}_{1} & 1 \\
-1 & 0
\end{array}\right), & i>1 \\
\left(\begin{array}{ll}
1 & 0 \\
0 & 1
\end{array}\right), & i=1\end{cases}
$$


Let $N$ be the total number of internal blow-ups on $\bar{Y}$. Let $\partial$ be the $2 \times(N+1)$ matrix with first column $\left(\begin{array}{l}0 \\ 0\end{array}\right)$ and subsequent columns defined by

$$
\left(\begin{array}{c}
-b_{i j} \\
a_{i j}
\end{array}\right), \quad j=1, \ldots, m,
$$

with each column repeated as many times as the number of internal blow-ups on the corresponding component $\bar{D}_{i j}$, i.e., $d_{i j}-\overline{d_{i j}}$. The following is a slight generalization of [M, Lemma (3.5) and succeeding discussion]:

(3.7) Proposition. There is a handle-body decomposition of $M:=Y \backslash N(D)$ consisting of two-handles $\left\{h_{0}^{2}, h_{1}^{2}, \ldots, h_{N}^{2}\right\}$, one-handles $\left\{h_{1}^{1}, h_{2}^{1}\right\}$, and a zerohandle such that the homology of $M$ is just the homology of the sequence

$$
0 \rightarrow \mathbb{Z}\left\langle h_{0}^{2}, h_{1}^{2}, \ldots h_{N}^{2}\right\rangle \stackrel{\partial}{\rightarrow} \mathbb{Z}\left\langle h_{1}^{1}, h_{2}^{1}\right\rangle \rightarrow 0 .
$$

Remark. From (3.6), $\partial$ has $(0,1)$ as a column. So $\pi_{1}(M)=\mathbb{Z}\left\langle h_{1}^{1}, h_{2}^{1}\right\rangle / \operatorname{im}(\partial)$ is always cyclic, as claimed in [LW].

It is possible to compute examples using this proposition. In particular, since the embedding dimension is precisely the length $n$ of the dual (3.1), for bounded embedding dimension the number of intermediate surfaces $\bar{Y}$ is also bounded, and all possible smoothings can be constructed by determining all the ways of blowing up from one of these to realize a given $D$. For each realization, Proposition (3.7) allows us to compute $\pi_{1}(M)=H_{1}(M)$. There are, in fact, 18 different $\bar{Y}$ of length equal to 7 , six of length 6 , two of length 5 , and one each of length 4 and 3 . Here is the list of (the negatives of) their self-intersection numbers:

\section{(3.8) TABLE}

$\begin{array}{llll}\text { Length }=3: & \text { Length }=6: & \text { Length }=7: \\ {[-1,-1,-1]} & {[3,0,-1,1,2,1]} & {[4,1,2,2,1,-1,0]} & {[2,1,2,0,1,1,2]} \\ & {[2,2,0,-1,2,1]} & {[3,2,1,3,1,-1,0]} & {[2,1,2,-1,1,1,3]} \\ \text { Length }=4: & {[2,1,2,0,0,1]} & {[3,1,3,1,2,-1,0]} & {[3,1,2,-1,0,3,1]} \\ {[-1,0,1,0]} & {[2,1,1,0,1,1]} & {[3,1,2,2,1,0,0]} & {[3,1,2,2,1,0,0]} \\ & {[3,1,1,-1,1,1]} & {[3,1,2,1,0,1,1]} & {[2,2,1,3,1,0,0]} \\ \text { Length }=5: & {[1,1,1,1,1,1]} & {[4,1,2,1,-1,1,1]} & {[2,1,3,1,2,0,0]} \\ {[1,1,2,0,-1]} & & {[3,1,2,2,-1,0,2]} & {[2,1,2,1,1,1,1]} \\ {[1,1,1,0,0]} & & {[2,2,1,3,-1,0,2]} & {[3,2,1,2,-1,1,1]} \\ & & {[2,1,3,1,0,2]} & {[3,1,1,0,1,2,1]}\end{array}$

Any $(Y, D)$ must arise from blowing up at interior points of one of these surfaces. It is first necessary to blow up sufficiently to make the cycle negativedefinite, so there is some mandatory blowing up on each of these cycles (see (1.2)). In each case the minimum required blowing-up is already sufficient to make the complement of $D$ simply-connected.

(3.9) Lemma. For a cusp singularity $(X, p)$ satisfying $\operatorname{Emb} \cdot \operatorname{dim}(X, p) \leq 7$, $\pi_{1}(M)=0$ for $M$ the Milnor fiber of any smoothing.

Proof. Any smoothing of a cusp satisfying the hypothesis must arise from Table (3.8). If a smoothing arises from a $(\bar{Y}, \bar{D})$ such that a pair of adjacent components $\bar{D}_{i}, \bar{D}_{i+1}$ both require internal blow-ups (i.e., $-\bar{D}_{k}^{2}<2, k=i, i+1$ ) then $\partial$ in (3.7) is surjective, implying the result. To see this, relabel the adjacent 
components as $\bar{D}_{1}$ and $\bar{D}_{2}$, and compute $\partial$ directly using (3.6):

$$
\left(\begin{array}{ll}
a_{1} & b_{1} \\
c_{1} & d_{1}
\end{array}\right):=\left(\begin{array}{ll}
1 & 0 \\
0 & 1
\end{array}\right) \text { and }\left(\begin{array}{ll}
a_{2} & b_{2} \\
c_{2} & d_{2}
\end{array}\right):=\left(\begin{array}{ll}
\bar{d}_{1} & 1 \\
-1 & 0
\end{array}\right) \text {. }
$$

From this, $\partial$ has the following form: $\partial=\left(\begin{array}{cccc}0 & 0 & -1 & \cdots \\ 0 & 1 & *\end{array}\right)$, which is clearly surjective. To finish the proof, note that every cycle in Table (3.8) has a pair of consecutive components satisfying $-\bar{D}_{k}^{2}<2$, thus requiring internal blow-ups.

The lemma's conclusion is not true for Emb. dim $>7$. A simple example is the following. Start with the length 7 cycle $[2,1,2,1,1,1]$, and blowup at a node so as to separate the components which satisfy $-\bar{D}_{i}^{2}<2$. One obtains a surface $(\bar{Y}, \bar{D})$ which blows up to a cusp smoothing with nontrivial $\pi_{1}$. Explicitly:

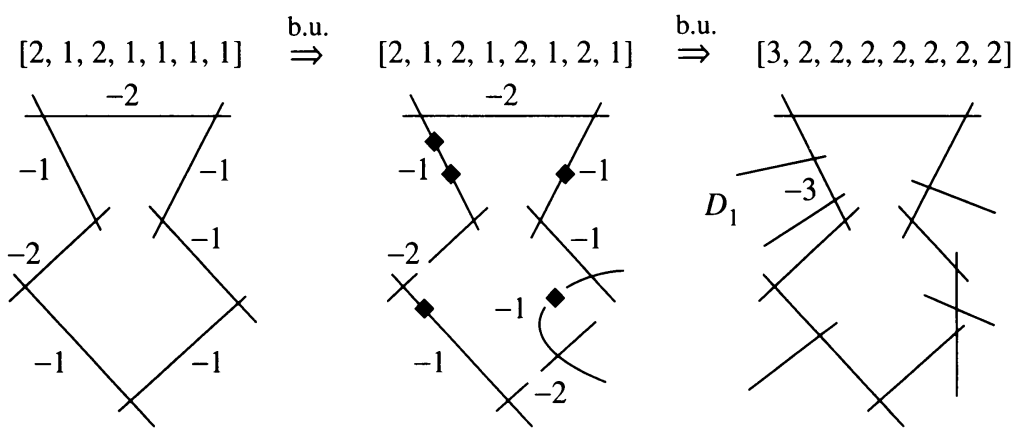

Using (3.6), $\partial=\left(\begin{array}{ccccc}0 & 0 & -2 & 0 & 2 \\ 0 & 1 & 1 & -1 & -1\end{array}\right)$ (redundant columns are omitted). So $\pi_{1}(Y \backslash D)=\mathbb{Z} / 2 \mathbb{Z}$.

The content of (3.9) is stated without proof in [LW, Theorem 6.6.1]; there it is also claimed that $\operatorname{Emb} \cdot \operatorname{dim}(X, p) \leq 8$ implies $\pi_{1}(M)=0$ or $\mathbb{Z} / 2 \mathbb{Z}$. It is clear from this lemma how to prove the other part of their claim, though it requires some calculation.

Proposition (3.7) also applies to simple-elliptic singularities. Any smoothing of a simple-elliptic $(X, p)$ is again obtained from an intermediate surface as in Table (3.8) but must satisfy the additional condition $-\bar{D}_{i}^{2}=2$. Thus, in particular,

(3.10) Lemma. For a simple-elliptic singularity $(X, p)$ satisfying Emb. $\operatorname{dim}(X, p) \leq 7, \pi_{1}(M)=0$ for $M$ the Milnor fiber of any smoothing

Proof. Same as for Lemma (3.9). As was true for cusps, $\pi_{1}(M)$ is in general a finite cyclic group. The example of the cusp smoothing of Emb. dim $=8$ with nontrivial $\pi_{1}$ can be adapted to give an example of a simple-elliptic smoothing with $\pi_{1}(M)=\mathbb{Z} / 2 \mathbb{Z}$ : Contract one of the exceptional curves passing through $D_{1}$ to obtain the cycle $[2,2,2,2,2,2,2,2]$. The calculation of its fundamental group is the same as for the cusp (more than one internal blow-up on a component contributes cycles to the second homology group, rather than destroying cycles in $H_{1}$ ).

(3.11) Not every smoothing of a degree 8 simple-elliptic has $\pi_{1}=\mathbb{Z} / 2 \mathbb{Z}$. If instead of blowing up $[2,1,2,1,1,1,1]$ symmetrically at the node between the 
third and fourth " 1 " to get the intermediate surface $[2,1,2,1,2,1,2,1]$, we blow up asymmetrically between the fourth and fifth "1" to get $[2,1,2,1,1$, $2,1,2]$, the result is a surface with two adjacent components requiring internal blow-ups. Therefore, when the internal blow-ups are performed to get the smoothing, its Milnor fiber is simply-connected, by the proof of Lemma (3.9). It is shown in [LW, (6.4)] that degree 8 simple-elliptics have five smoothing components and that degree 9 simple-elliptics have nine smoothing components. It appears that the fundamental group of $M$ can distinguish two such components for degree 8 simple-elliptics.

(3.12) It is well known that a simple-elliptic $(X, p)$ has a smoothing if and only if its degree $(=\mathrm{Emb} . \mathrm{dim})$ is at most nine (see [LW, 6.4]). For degree 8, the only possibilities for $\pi_{1}(M)$ are 0 and $\mathbb{Z} / 2 \mathbb{Z}$. For degree 9 , the group $\mathbb{Z} / 3 \mathbb{Z}$ appears. The intermediate surface that blows up to the degree 9 smoothing is $[2,1,2,2,1,2,2,1,2]$. It is constructed by blowing up $[1,1,1,1,1,1]$ symmetrically at the three nodes between the first and second, third and fourth, and fifth and sixth components. Using (3.6), $\partial$ is the map $\partial=\left(\begin{array}{cccc}0 & 0 & -3 & 3 \\ 0 & 1 & 1 & -2\end{array}\right)$. The quotient by the image of $\partial$ is $\mathbb{Z} / 3 \mathbb{Z}$. There is no other way to realize a length 9 cycle of $(-2)$ rational curves on a rational surface, so this is the only group that occurs for a simple-elliptic smoothing of degree 9.

\section{REFERENCES}

[GS] G.-M. Greuel and J. H. M. Steenbrink, On the topology of smoothable singularities, Proc. Sympos. Pure Math., vol. 40, part 1, Amer. Math. Soc., Providence, RI, 1983, pp. 535-545.

[K] K. Kodaira, On compact analytic surfaces. II, III. Ann. of Math. (2) 77 (1963), 563-626; 78 (1963), 1-40.

[L1] E. Looijenga, The smoothing components of a triangle singularity. I, Proc. Sympos. Pure Math., vol. 40, part 2, Amer. Math. Soc., Providence, RI, 1983, pp. 173-184.

[L2] _ The smoothing components of a triangle singularity. II, Math. Ann. 269 (1984), 357387.

[L3] _ Rational surfaces with an anti-canonical cycle, Ann. of Math. (2) 114 (1981), 267-322.

[LW] E. Looijenga and J. Wahl, Quadratic functions and smoothing surfaces singularities, Topology 25 (1986), 261-291.

[M] L. J. McEwan, Families of rational surfaces preserving a cusp singularity, Trans. Amer. Math. Soc. 321 (1990), 691-716.

[MP] R. Miranda and U. Persson, Configurations of $I_{n}$ fibers on elliptic K3 surfaces, Math. Z. 201 (1989), 339-361.

[P] H. Pinkham, Smoothings of the $D_{p q r}$ singularities, $p+q+r=22$, Proc. Sympos. Pure Math., vol. 40, Amer. Math. Soc., Providence, RI, 1983, pp. 373-377.

[S] T. Shioda, On elliptic modular surfaces, J. Math. Soc. Japan 24 (1972), 20-59.

[SI] T. Shioda and H. Inose, On singular K3 surfaces, Complex and Algebraic Geometry (papers dedicated to Kodaira) (W. L. Baily and T. Shioda, eds.), Cambridge Univ. Press, London, 1977.

Department of Mathematics, The Ohio State University at Mansfield, Mansfield, Оніо 44906

E-mail address: mcewan@function.mps.ohio-state.edu 